\title{
Helium line ratio spectroscopy for high spatiotemporal resolution plasma edge profile measurements at ASDEX Upgrade ${ }^{a)}$
}

\author{
M. Griener, ${ }^{1}, 2$, b) E. Wolfrum, ${ }^{1}$ M. Cavedon, ${ }^{1}$ R. Dux, ${ }^{1}$ V. Rohde, ${ }^{1}$ M. Sochor, ${ }^{1}$ J.M. Muñoz Burgos, ${ }^{3}$ O. \\ Schmitz, ${ }^{4}$ U. Stroth, ${ }^{1,2}$ and the ASDEX Upgrade Team ${ }^{\text {c) }}$ \\ 1) Max Planck Institute for Plasma Physics, Boltzmannstr. 2, 85748 Garching, \\ Germany \\ ${ }^{2)}$ Physik Department E28, Technische Universität München, 85748 Garching, \\ Germany \\ 3) Astro Fusion Spectre, astrofusionspectre@gmail.com \\ ${ }^{4)}$ Department of Engineering Physics, University of Wisconsin-Madison, USA
}

(Dated: 22 May 2018)

The thermal helium beam edge diagnostic has recently been upgraded at the ASDEX Upgrade (AUG) tokamak experiment. Line ratio spectroscopy on neutral helium is a valuable tool for simultaneous determination of the electron temperature and density of plasmas. The diagnostic now offers a temporal resolution of $900 \mathrm{kHz}$ with a spatial resolution of up to $3 \mathrm{~mm}$ at 32 lines of sight (LOS) simultaneously. The LOS cover a radial region of $8.5 \mathrm{~cm}$, starting at the limiter radius and reaching into the confined region beyond the separatrix. Two components are of particular importance for the aforementioned hardware improvements. The first is the optical head, which collects the light from the experiment. Equipped with an innovative clamping system for optical fiber ends, an arbitrary distribution pattern of LOS can be achieved to gain radial and poloidal profiles. The second major development is a new polychromator system that measures the intensity of the $587 \mathrm{~nm}, 667 \mathrm{~nm}, 706 \mathrm{~nm}$, and $728 \mathrm{~nm}$ helium lines simultaneously for 32 channels with filter-photomultiplier tube arrays.

Thus, the thermal helium beam diagnostic supplements the AUG edge diagnostics, offering fast and spatially highly resolved electron temperature and density profile measurements that cover the plasma edge and scrapeoff layer region. Plasma fluctuations, edge localized modes, filaments, and other turbulent structures are resolved, allowing analysis of their frequency and localization or their propagation velocity.

PACS numbers: 52

Keywords: plasma edge diagnostics, helium line ratio spectroscopy, optical head, wire eroding, polychromator, photo multiplier array, interference filter, electron temperature, electron density

\section{INTRODUCTION}

Line ratio spectroscopy on neutral helium $\left({ }^{2-4}\right.$ and references therein) is a valuable tool for simultaneous determination of the electron temperature $T_{\mathrm{e}}$ and density $n_{\mathrm{e}}$ of plasmas. Good spatial resolution can be achieved for spectroscopic diagnostics for large plasma devices used in fusion research, such as tokamaks or stellerators, by means of active spectroscopy. This means that neutral helium can be actively injected into the plasma as a beam ${ }^{3}$ or collimated cloud ${ }^{5}$ to achieve high local densities. The injected helium is excited mainly by collisions with the plasma electrons and therefore emits light. The emitted light at the intersection of the helium beam and the lines of sight (LOS) is collected by an optical head, focusing it onto optical fibers. The background signal can be subtracted by chopping the

\footnotetext{
a) Invited paper published as part of the Proceedings of the 22nd Topical Conference on High-Temperature Plasma Diagnostics, San Diego, California, April, 2018.

b) Electronic mail: michael.griener@ipp.mpg.de

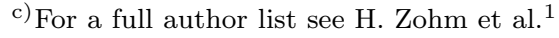

helium injection.

Helium line ratio spectroscopy is based on the simultaneous intensity measurement of at least three neutral helium (He I) lines. The Grotrian diagram of He I is divided into singlet $(\mathrm{s})$ and triplet $(\mathrm{t})$ states. The functional principle of the diagnostic relies on the differing temperature and density behaviours of $\mathrm{s} / \mathrm{s}$ and $\mathrm{s} / \mathrm{t}$ line intensity ratios. Ratios between two singlet lines are commonly used to calculate the electron density, whereas electron temperature sensitivity is achieved by taking $\mathrm{s} / \mathrm{t}$ ratios for the calculation. The comparison of the measured ratios of suitable lines ${ }^{4}$ with a collisional radiative model ${ }^{6}(\mathrm{CRM})$ enables the reconstruction of the underlying values for electron density and temperature.

For the simultaneous measurement of several He I line intensities, a broad-band spectrometer equipped with a CCD camera can be used ${ }^{7}$. This also enables the observation of other spectral lines, which may disturb the exclusive measurement of He I lines. The disadvantage of this approach is that typical spectrometers end up with a poor time resolution on the order of $1-100 \mathrm{~ms}$. To perform measurements a thousand times faster, a system based on photomultiplier tubes (PMTs) ${ }^{8,9}$ or avalanche diodes ${ }^{10}$ has to be used. Two approaches are common 
for the spectral selection of He I lines, namely, either the usage of a spectrograph ${ }^{11}$ or the combination of beam splitters and filters. Within this approach, the light from each fiber is split with semipermeable ${ }^{9}$ or dichroic mirrors ${ }^{8}$ into different optical paths, where the desired $\mathrm{He}$ I line is selected with a narrow-band interference filter. Typically, such polychromator systems, which are often called filterscopes ${ }^{2,8}$, are designed to measure the light from only one optical fiber. This results in a large number of required filter systems, every single one of which has to be modified if another wavelength should be measured. More flexibility can be obtained by combining all spatial channels within one device using PMT arrays. This is realized in the setup described in reference ${ }^{3}$, for which a direct optical access to the measurement region as a 2 - $\mathrm{D}$ image is required.

This paper describes the hardware improvements to the thermal helium beam system at ASDEX Upgrade (AUG), which enables temporally and spatially highly resolved measurements with a $900 \mathrm{kHz}$ sample rate and a spatial resolution up to $3 \mathrm{~mm}$. The hardware system is based on an in-vessel optical head, which transmits the light by optical fibers to a four-color, 32-channel polychromator system.

The paper is organized as follows: in section II, the invessel components are introduced, including the piezoelectric valve and the optical head, paying special attention to the fiber-clamping system. In section III, a detailed description of the newly developed polychromator system is given. This covers the lenses for imaging, the dichroic mirrors, and the narrow-band interference filters as well as the PMT arrays and the data acquisition system used for the fast diagnostic. Selected measurements demonstrating the high spatiotemporal resolution of the He-beam diagnostic are presented in section IV.

\section{IN-VESSEL COMPONENTS}

The in-vessel components for active line ratio spectroscopy on helium at AUG are depicted in Figure 1, which shows a CAD-based view of sector 13 in the toroidal direction.

The piezoelectric valve for helium injection is placed at the magnetic low-field side (radius $R=2.19 \mathrm{~m}$ and height $z=-0.16 \mathrm{~m}$ with respect to the midplane, cf. Figure 4). The valve is equipped with a $0.4 \mathrm{~mm}$ thick capillary to inject cone-shaped gas clouds with a halfopening angle of $20^{\circ}$ into the scrape-off layer (SOL). Regulated by the filling pressure of the valve, gas fluxes between $10^{18} \mathrm{part} / \mathrm{s}$ and $5 \times 10^{21} \mathrm{part} / \mathrm{s}$ can be achieved. The gas flux can be modulated with switch on/off times of $\leq 0.5 \mathrm{~ms}$. A detailed description of the valve can be found in reference ${ }^{5}$. The necessary amount of diagnostic gas of around $10^{19}$ part/s does not seem to influence the local values of $n_{\mathrm{e}}$ and $T_{\mathrm{e}}$ as local deuterium emission is not influenced by the helium puffs (see also $\left.{ }^{5},{ }^{4}\right)$.
The emitted light is collected by the optical head or a dip tube which provides a 2-D image of the gas cloud. The image is transmitted by an image conductor to the fast camera "Phantom v711" which can be equipped with a filter for one specific wavelength or record the total emission of the cloud. The information extracted from a full 2-D image can be used for gas-puff imaging (GPI) (cf. ${ }^{12,13}$ ), allowing turbulent blob structures in the SOL to be traced.

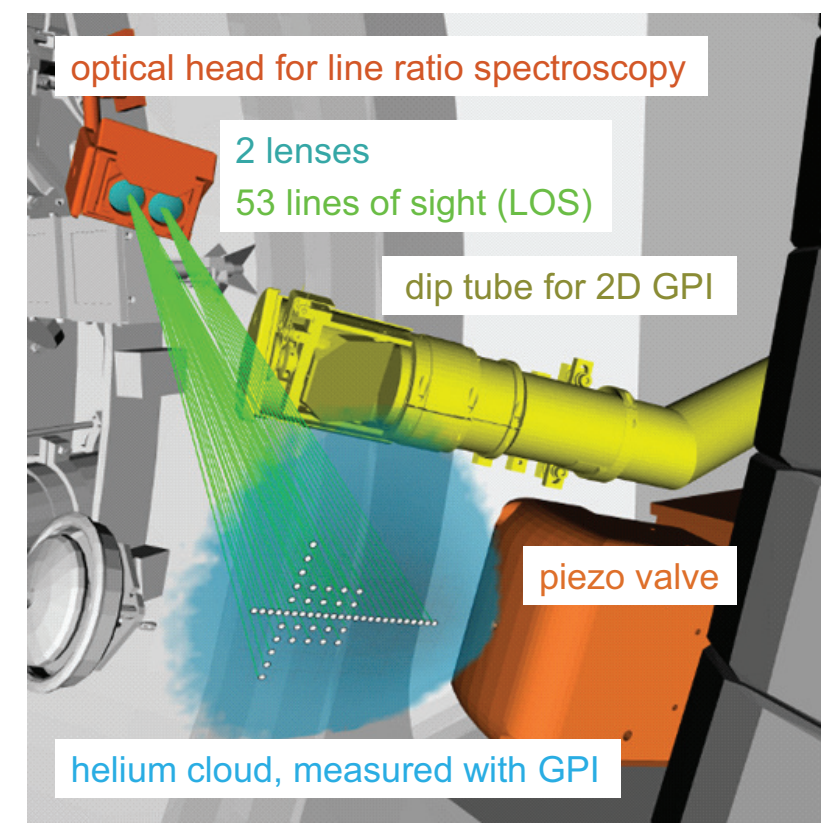

FIG. 1: View in the toroidal direction, representing the helium beam and GPI diagnostics at AUG. A detailed representation of the LOS is given in Figure 4.

The newly developed two-lens optical head for helium line ratio spectroscopy provides 52 LOS. The optical head is equipped with an innovative clamping system for optical fiber ends (cf. Section IIB). With this technique, an arbitrary distribution pattern of LOS can be achieved to gain radial and poloidal profiles. In our case, two perpendicular 1-D arrays offer profiles in the radial and poloidal directions, whereas a 2 -D matrix of $5 \times 5$ fibers allows the tracing of turbulent blob structures in the SOL. Connected with the polychromator system (cf. Section III) the optical head delivers the electron density and temperature values of the measurement points, which can be superposed with the 2-D image from the GPI.

The two lenses of the optical head have a distance of $d=0.69 \mathrm{~m}$ to the helium injection point. With a clear aperture of $34 \mathrm{~mm}$ (area $A=9.1 \times 10^{-4} \mathrm{~m}^{2}$ ), one lens covers a solid angle of $\Omega=A / d^{2}=1.9 \times 10^{-3} \mathrm{sr}$.

The light collected by the lenses is coupled to in-vessel fibers, placed at a distance of $88 \mathrm{~mm}$ behind the lenses. The numerical aperture (NA) of the fibers is 0.22 . This corresponds to a light acceptance angle of $12.7^{\circ}$, such that all light collected by the lenses is accepted by the optical fibers. This, as well as the schematic structure of 
the optical head, is presented in Figure 2.

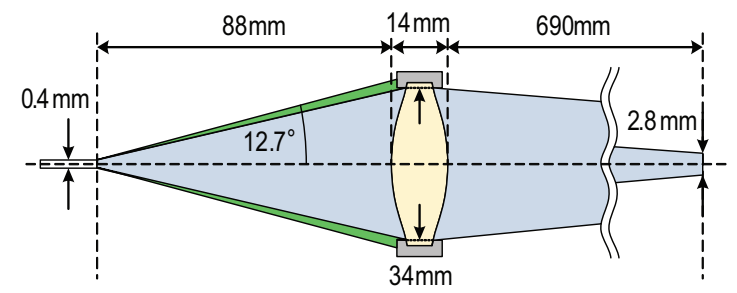

FIG. 2: Schematic representation of the optical head. The lens (shaded in yellow) collects the light along the LOS (shaded in blue) with a diameter of $2.8 \mathrm{~mm}$ at the narrowest point. The light is bundled into the bare end of optical fibers with a $0.4 \mathrm{~mm}$ core diameter and an NA of 0.22 . The NA, which determines the acceptance angle of the fiber is illustrated as the green-shaded area.

A detailed view of the optical head is given in Figure 3. The fiber ends in the interior of the optical head are placed as a 2-D-pattern in a wire-eroded holder as described in section II B. The head is equipped with two aspherical lenses, "AFL50-80-S-A2" 14, with a nominal focal length of $80 \mathrm{~mm}$ (effective focal length, EFL $=85 \mathrm{~mm}$ ) and a diameter of $35 \mathrm{~mm}$. The lenses are protected by the normally closed magnetic shutter in between discharges. Two lenses are used to increase the spatial resolution of the diagnostic, since this reduces the maximum angle between the LOS and the field lines at the observation point as explained in section II A.

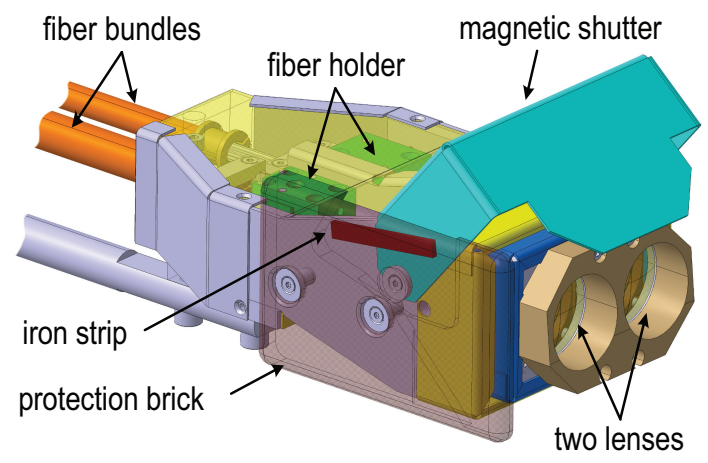

FIG. 3: CAD drawing of the two-lens optical head including the magnetic shutter in an open position with the iron strip (red) and the optical fiber holder in the head's interior. The two fiber bundles are connected to a vacuum feed through.

\section{A. Spatial resolution}

The spatial resolution of active beam spectroscopy depends on three major factors; beam width, the alignment of the LOS and the beam orientation with respect to the magnetic field lines, and the diameter of the LOS at the observation point. The latter factor influences the amount of collected light, so a diameter of $3 \mathrm{~mm}$ is chosen. This sets the lower limit for the spatial resolution, as measured in units of $\mathrm{mm}$. The best resolution can be achieved if the beam is directed perpendicularly to the flux surfaces and the LOS are tangential to the flux surfaces in the region of intersection with the beam. This perfect alignment is, of course, only possible for one LOS per lens. The angle between the remaining LOS and the flux surfaces increases with the radial region covered by one lens. To achieve a good resolution over a radial distance of $8.5 \mathrm{~cm}$, which covers the whole SOL, two lenses are used in the optical head.

The emission of the helium cloud along the path of each LOS is calculated with a forward model such that a specific measurement location can be assigned to each LOS. The points of maximal emission along each LOS are marked by red crosses in Figure 4. The radial intervals, which cover $68 \%$ of the emission along a LOS, are set as the radial errors of the signals, respectively.

The worst resolution of all LOS corresponds to the innermost LOS, where the helium beam is broadest and the LOS has the steepest angle toward the field lines. This broadening corresponds to a spatial range of $3 \mathrm{~mm}$, which has to be added to the $3 \mathrm{~mm}$ resolution minimum given by the LOS diameter. The radial broadening for the best-aligned LOS is negligible whereas the mean value is $0.9 \mathrm{~mm}$.

\section{B. Fiber holder}

The most innovative part of the optical head is the mechanism that fixes the fiber ends in the inside of the optical head. The presented system allows the manufacturing of holders by wire eroding for arbitrary LOS patterns, which are images of the fiber distribution. The optical head is a modular system, such that the fiberequipped fiber holder for each lens can be mounted separately in the head's interior. This is necessary for installing the optical head as the fibers are part of optical feed through and thus mounting on site is needed. The two fiber holders pictured in Figure 5 are not symmetric. Holder 1 is equipped with a 2-D array, while holder 2 contains only a single row of fibers to increase the measurement range for the radial profiles.

Both fiber holders are based on a clamping system, which fixes the fiber ends reversibly. Without using of any glue, the system is well suited to usage in vacuum chambers, including the baking procedure at $150{ }^{\circ} \mathrm{C}$.

The high-OH (high concentration of hydroxyl groups) "UV400-440-480P" 15 fibers with NA $=0.22$ and an outer diameter of $0.480 \mathrm{~mm}$ were used in-vessel. The holders were wire-eroded from an aluminum block with the "Sodick AG400L" eroding machine equipped with a $0.15 \mathrm{~mm}$ brass wire. This enabled the cutting of single wire channels for the optical fibers. The 3-D arrangement of the fibers is built in such a way that every fiber is aligned toward the center of the corresponding lens (cf. Figure 6). 


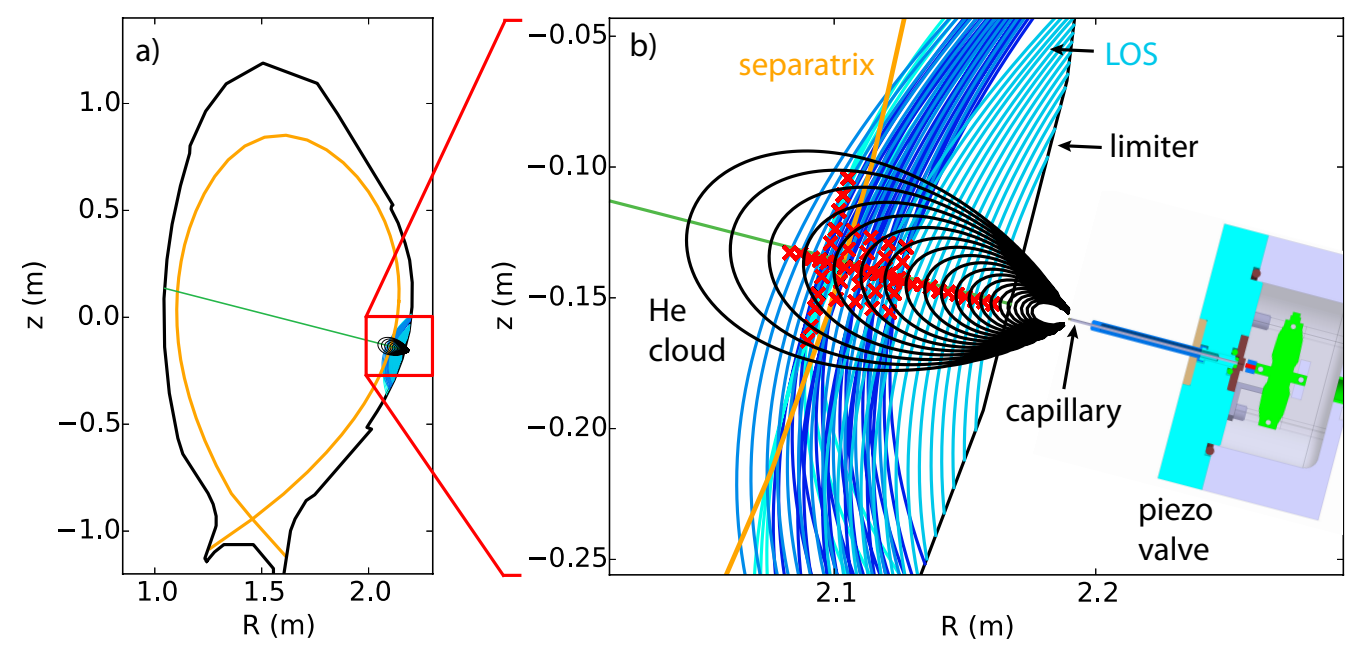

FIG. 4: Poloidal cross section of AUG. The LOS (blue), the piezo valve, and a contour plot of the helium density (black), as well as the separatrix (orange) and limiter position (black) are illustrated. The green line shows the central axis of the helium cloud, which lies perpendicular to the flux surfaces and the LOS at the measurement region. The red crosses mark the points of highest emission along each LOS.

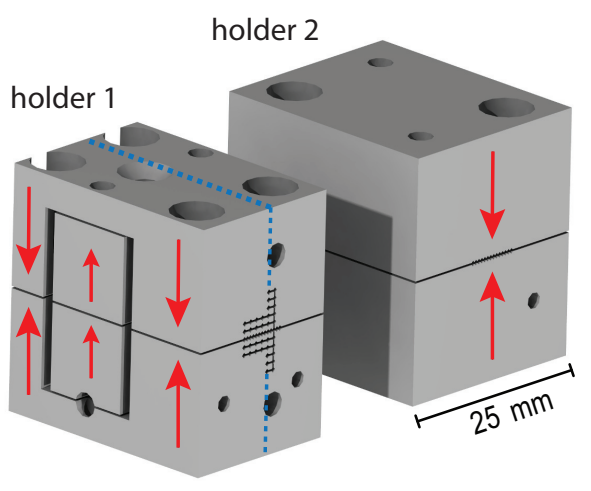

FIG. 5: CAD drawing of the fiber holder manufactured by wire eroding out of an aluminum block. The arrows represent the direction of tension used to fix the fibers mechanically in their positions. A cross-sectional view of the dashed marked plane is given in Figure 6 .

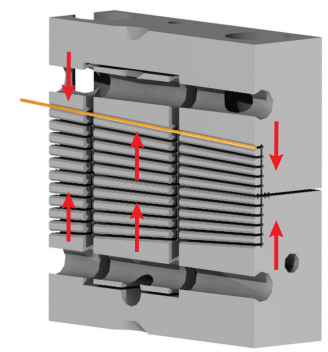

FIG. 6: CAD cut of the fiber holder demonstrating the arrangement of fiber channels in the block's interior. The red arrows represent the direction of tension used to fix the fibers mechanically in their positions. One

fiber is illustrated in the uppermost channel.
This minimizes the maximum angle of incidence of the light with respect to the fiber and enables an optimal light incoupling.

The reversible fixing of the fibers in the 2-D pattern is achieved by shearing the inner part of the holder with respect to its front and back sides by simultaneously pressing the holder's upper and lower parts together, as illustrated in Figure 5 with red arrows. For the 1-D pattern, clamping by pressing both holed parts together is sufficient.

\section{POLYCHROMATOR SYSTEM}

The light collected by the optical head in the torus is transmitted from the experimental hall to the laboratory by optical fibers of $52 \mathrm{~m}$ length, $0.4 \mathrm{~mm}$ core diameter, and an NA of 0.22. In the laboratory, the light is coupled to a fiber bundle with an included taper (cf. Section III A). Its 32 round end caps are optically mapped by a telecentric lens to the photomultiplier arrays. As illustrated in Figure 7, the four detectors are therefore all placed at the same distance of $20.5 \mathrm{~cm}$ with respect to the end lens of the objective.

On the way to the detector, the light of each fiber is split into four wavelength parts to measure the $587.6 \mathrm{~nm}$, $667.8 \mathrm{~nm}, 706.5 \mathrm{~nm}$ and $728.1 \mathrm{~nm}$ helium lines simultaneously. The splitting of the spectral components is achieved using dichroic mirrors and the subsequent filtering with high-transmission interference filters (cf. Section III B) placed directly in front of the detector plane. To match the elongated sensitive area of the detectors (cf. Section III C), a cylindrical lens is placed behind the end lens of the objective to elongate the image of the fibers.

High-transmission optics in combination with fast- 
responding PMT detectors and appropriate analog digital converters for the PMT output voltage allow a temporal resolution of $900 \mathrm{kHz}$. This makes the newly designed setup an excellent device for high resolution helium line ratio spectroscopy, also offering a compact design and a high flexibility in changing the selected wavelengths.

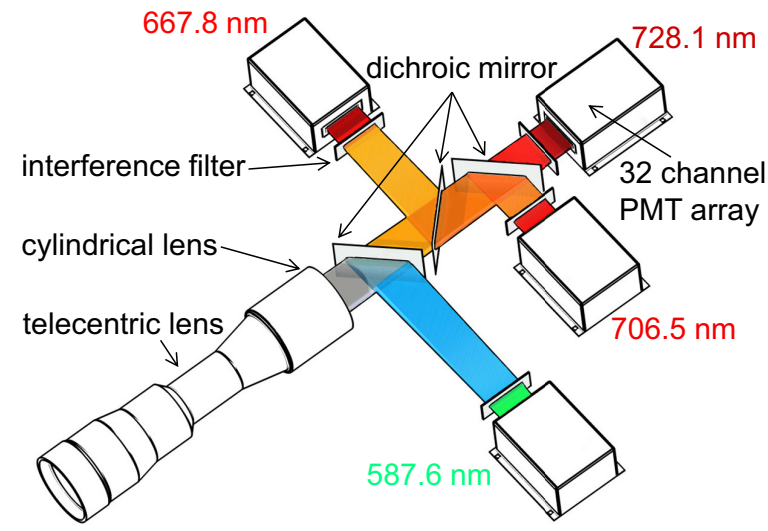

FIG. 7: Schematic representation of the 32-channel polychromator system, which includes imaging optics (left) that relay the light from the fiber array (left of the objective, not pictured) to the detectors. The light of each channel is divided into four wavelengths and measured separately.

\section{A. Fiber bundle and telecentric lens}

a. Fiber bundle The fibers delivering light from the experimental hall are connected by fiber-optic connectors (FC) to a bundle ${ }^{16}$ of 32 fibers. At the end of the bundle, the fibers with a core diameter of $0.8 \mathrm{~mm}$ are placed next to one another with a spacing of $1 \mathrm{~mm}$ in a linear array. This geometry matches the detector geometry (cf. Section III C), such that a one-to-one mapping is possible. Each fiber of the bundle is tapered from a $0.4 \mathrm{~mm}$ core diameter on the FC side to a $0.8 \mathrm{~mm}$ core diameter on the imaging side. This process, which increases the diameter linearly, preserves the etendue of the fibers. The etendue $\mathcal{E}$ is defined with the fiber diameter $d$ as

$$
\mathcal{E}=\frac{\pi^{2}}{4} d^{2} \mathrm{NA}^{2}
$$

It can be seen directly that doubling the diameter halves the NA. The resulting NA of 0.11 corresponds to a light opening angle of $6.3^{\circ}$ compared to an angle of $12.7^{\circ}$ for $\mathrm{NA}=0.22$, measured toward the optical axis. This process increases the transmittance of light through the telecentric lens.

b. Telecentric lens The main boundary condition when designing a filter based system is that the light which passes the filters and mirrors should be as collimated, i.e. parallel, as possible. In general, this can be achieved by placing the fiber ends at the focal distance $f_{0}$ of a collimator lens and then focusing the light after wavelength splitting and filtering by an ocular lens to the detectors. Doing this, the light from off-axis fibers with a distance $r_{0}$ to the optical axis shows an angle of $\arctan \left(r_{0} \cdot f_{0}^{-1}\right)$ to the optical axis after the collimator lens. The high distance between the collimator and ocular lenses needed to split the four wavelength parts would lead to unreasonably high diameters for the ocular lenses. Therefore, most systems are designed to only handle a small number of fibers close to the optical axis. With the disadvantage of losing light, this problem can be solved using a telecentric lens as presented below.

Telecentric lenses produce an orthographic view of the subject. This is relevant because, with this technique, the distance between the off-axis rays and the optical axis stays constant. This major advantage comes at the cost of loss of light at the built-in aperture of the lens, which can only be passed by beams that make a small angle with the optical axis.

In the simplest case, a telecentric lens consists of two lenses with the same focal length $f$, separated by a distance of $2 f$ with an aperture placed between them. Light passing through the lens at an angle higher than the teletricity angle toward the optical axis is blocked by this aperture. Therefore, more light is transmitted through the lens when small-NA fibers compared to highNA fibers are used.

If both objective lenses have the same focal length, a one-to-one image is produced. In this case, the position of the telecentric lens between the object and its image does not influence the image. A sharp image is achieved if the distance between the object and the image plane is equal to the length of the telecentric lens plus two times its effective focal length. For the presented polychromator system, the telecentric lens TC16M036 ${ }^{17}$ is used. Its length is $250 \mathrm{~mm}$ and its symmetric end lenses show a diameter of $51 \mathrm{~mm}$. The effective focal length of the objective is $f=102.6 \mathrm{~mm}$. As the optical fibers are directly placed at the surface of the entrance lens, the distance between the exit lens of the objective and all detectors must be $205.2 \mathrm{~mm}$. To enable the exact positioning of the fiber bundle close to the first lens, the delivered F-mount of the telecentric lens was unscrewed and replaced by a custom made fiber adapter.

\section{B. Interference filters and dichroic mirrors}

The light of each channel is split with three dichroic mirrors in four wavelength intervals. The $50 \%$ cuton wavelengths of the mirrors are $655 \mathrm{~nm}, 695 \mathrm{~nm}$, and $718 \mathrm{~nm}$. To select the required wavelength for each photomultiplier array, small-band interference filters ${ }^{18}$ with full width at half maximum $<1.7 \mathrm{~nm}$ of the transmission band are used. The central wavelength $\lambda_{0}$ of the filters at normal incidence were measured to be $(587.4 \pm 0.1) \mathrm{nm}$, $(668.0 \pm 0.1) \mathrm{nm},(706.3 \pm 0.1) \mathrm{nm}$ and $(728.1 \pm 0.1) \mathrm{nm}$. Both, dichroic mirrors as well as interference filters with 
an effective reflection index $n_{\text {eff }}$ rely on stacked FabryPerot resonant cavities. The transmitted wavelength depends on the light's incident angle $\theta$, described by

$$
\lambda_{\theta}=\lambda_{0} \sqrt{1-\frac{\sin ^{2} \theta}{n_{\mathrm{eff}}^{2}}},
$$

which is a valid description up to some tens of degrees, as verified by measurements. The knowledge of the angle dependent wavelength shift is of specific interest for estimating the required alignment tolerances in the optical setup. In this case, the wavelength shift is $<0.5 \mathrm{~nm}$ for an alignment uncertainty of \pm 4 degree.

The dichroic mirrors are specified for an angle of incidence of $45^{\circ}$, where Equation 2 is no longer valid. Figure 8 shows the wavelength shift of the mirror with a $718 \mathrm{~nm}$ cut-on wavelength as a function of the incident angle. The wavelength of maximal transmission was measured in the transmission band. In the region around $45^{\circ}$, the wavelength shift is a linear function of the incident angle with a slope of $(1.89 \pm 0.06) \mathrm{nm} /$ degree. At $45^{\circ}$ the transmission reaches its maximum of $98 \%$ at a wavelength of $727.5 \mathrm{~nm}$, which makes precise alignment of the mirrors crucial. Within the setup presented, an alignment uncertainty including light divergence of $<1.5^{\circ}$ is ensured.

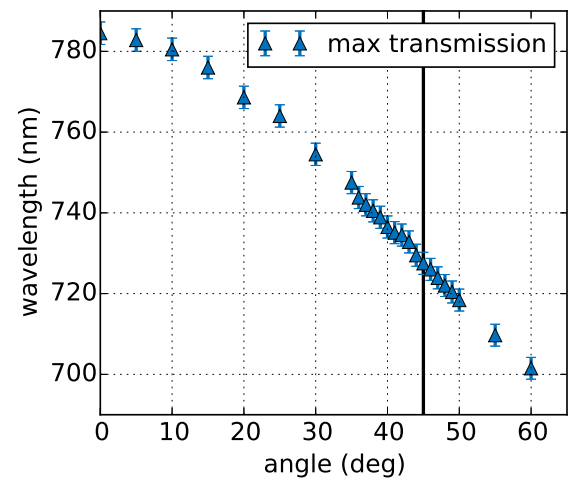

FIG. 8: Shift of the starting wavelength for the saturated region of the transmission band for the dichroic mirror with a $718 \mathrm{~nm}$ cut-on wavelength.

Figure 9 shows the transmission spectra of the dichroic mirrors compared to the position of the interference filters. The absolute transmission of the filters is not representative because the resolution of the spectrometer used (AvaSpec-2048x14-USB2 ${ }^{19}$ ) is on the order of the FWHM of the filters.

In contrast to systems where semipermeable mirrors would lead to a transmittance of light $<25 \%$ for each channel, the dichroic mirrors and filters were chosen in a way that guarantees the maximal transmittance for each of the four measured lines. With this setup, a portion of $>94 \%$ of the light passing the objective reaches the detectors.

By changing the filter in front of the first PMT array, the $\mathrm{He}^{+}$line at $468.6 \mathrm{~nm}$ can be observed alternatively to the $587 \mathrm{~nm}$ line.

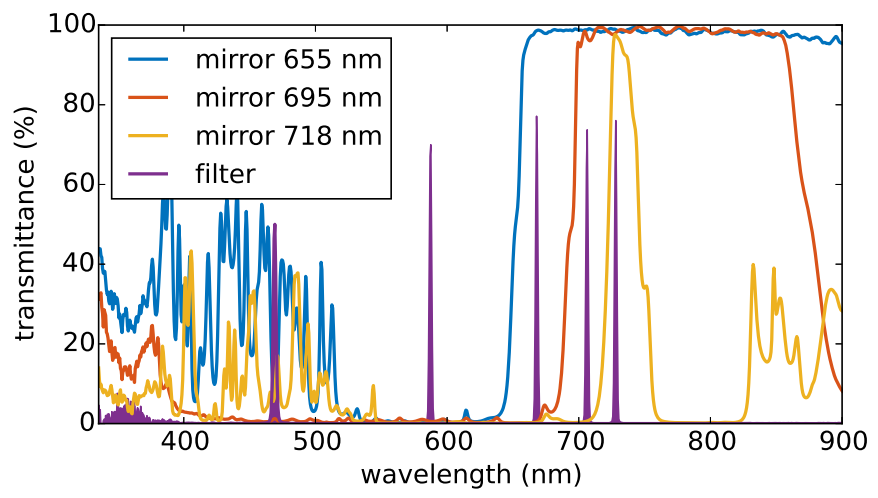

FIG. 9: Spectra of the three dichroic mirrors labeled with their cut-on wavelength compared to the transmission region of the interference filters.

\section{Detectors and data acquisition}

Light intensity is of prime importance in choosing an appropriate detection technique. Taking all transmission losses into account, the estimated photon flux lies in the range between $10^{7} \mathrm{~s}^{-1}$ and $10^{10} \mathrm{~s}^{-1}$, depending on the chosen emission line and the underlying plasma temperature and density. Especially for low photon fluxes, which limit the time resolution of the diagnostic, the best signal-to-noise ratio can be achieved with photomultiplier tubes, as explained in ${ }^{10}$.

To enable simultaneous measurement of several channels in one system, PMT array sensors with 32 channels (Hamamatsu $^{20}$ type H7260-20) are chosen. The detector is identical to the system previously used $\mathrm{in}^{3}$. In contrast to ${ }^{3}$, where the supply and amplification of the PMT array had to be added externally, linear multianode photosensor modules of the type H11460-20 with builtin high voltage power supplies and amplifiers are used. The compact detectors are shown in Figure 10 with and without an attached interference filter.

The photosensitive areas of each channel have dimensions of $0.8 \times 7 \mathrm{~mm}$, spaced by $1 \mathrm{~mm}$ as shown in Figure 11. The optical system, described in section III A, is used to image the fiber array onto this area in a one-toone representation. The elongation of the image of the round $0.8 \mathrm{~mm}$ fibers to $5.7 \mathrm{~mm}$ is achieved with a cylindrical lens with a $-500 \mathrm{~mm}$ focal length, placed $200 \mathrm{~mm}$ in front of the detector plane. This leads to homogeneous illumination of the detector area as represented in Figure 11. It can be seen that the single images of the fibers are well separated on the optical axis, whereas chromatic aberrations of the lens are visible for the side channels. The resulting optical crosstalk (up to $\approx 4 \%$ ) between the channels can be corrected using the calibration procedure discussed in section III D. 


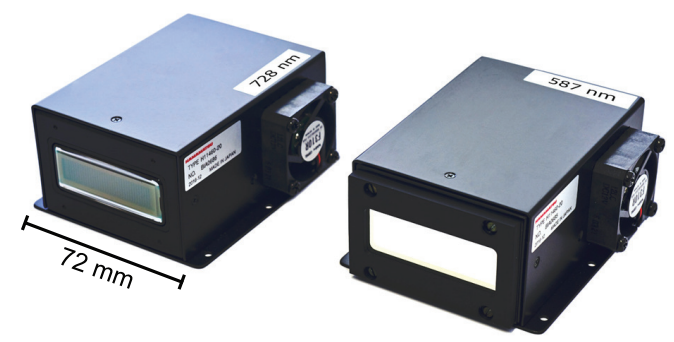

FIG. 10: Two Hamamatsu PMT array modules H11460-20 without (left) and with (right) an attached interference filter. The detector geometry is shown in Figure 11.

a)

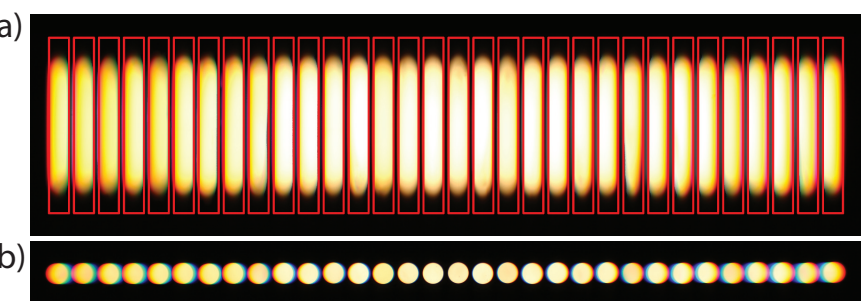

FIG. 11: Images of the fiber array at the detector plane. The images of the 32 single fibers are spaced by $1 \mathrm{~mm}$.

The round one-to-one image of the $0.8 \mathrm{~mm}$ fiber ends b) is elongated by the $f=-500 \mathrm{~mm}$ cylinder lens to

$5.7 \mathrm{~mm}$ a) to match the detector geometry. The sensitive areas of the PMT array are represented by the $0.8 \times 7 \mathrm{~mm}$ red rectangles.

For operation of the PMT modules, power supplies (Kniel: CAD 15.1,6) with $\pm 15 \mathrm{~V}$ and control voltages between $2 \mathrm{~V}$ and $3.2 \mathrm{~V}$ are used. The control voltage regulates the high voltage for the PMT dynodes between $500 \mathrm{~V}$ and $800 \mathrm{~V}$, leading to a maximal gain of $1 \times 10^{6}$ and a relative amplification of the output voltage by a factor of $\approx 60$ between the lowest and highest gains. The target output voltage lies in the range of $0-0.6 \mathrm{~V}$.

The data were recorded by a SIO-2 (serial input-output, cf. $^{21,22}$ ) data acquisition system using 32 analog-todigital converter (ADC) cards with four channels, respectively. The maximum sampling rate is $900 \mathrm{kHz}$ with a 14 Bit resolution. Two DAC units with two channels, respectively, are used to provide the control voltage for the PMT modules. Furthermore, the SIO-2 interface card has an additional feature providing connectivity to the experiment timing system as described $\mathrm{in}^{21}$. This allows time-stamp and trigger functionality for data acquisition. For one plasma discharge with a data acquisition time of $10 \mathrm{~s}$ around $2.1 \mathrm{~GB}$ of data are recorded.

\section{Calibration of the detection system}

To assign the output voltage of each channel of the PMT arrays to the detected line radiance, several calibration steps are necessary. On the one hand, absolute calibration of the system is not necessary, as constant factors like the aperture of the first lens and the LOS diameter cancel out when the ratios between two wavelengths are built. This also eliminates the influence of the helium flux upon the absolute emission strength. On the other hand, the relative calibration between PMTs is crucial. As a first calibration step, the DC offset of the PMTs and ADCs is subtracted from the signal and the crosstalk between the channels is compensated. Furthermore, the wavelength specific transmission of all optical elements, as well as the wavelength and channel specific quantum efficiencies of the PMT arrays, have to be corrected and different values of the PMT gain settings have to be compensated. This is done by calibrating the output voltage with a helium lamp with known line intensities. By chopping the helium beam during the plasma discharge (cf. Figure 12), the dynamic signal background, e.g. by bremsstrahlung, is subtracted from the active signal of the helium beam.

One uncertainty in the calibration of most spectroscopic diagnostics in fusion research arises from possible coatings on the first lens, which is located close to the plasma in the vacuum chamber. These coatings can change during the operational campaign, whereas in-vessel calibrations are only possible during the maintenance periods of the device. By measuring the transmission before and after the experimental campaign, the influence of surface layers upon lenses can mostly be estimated. However, the data presented in section IV may still contain some remaining uncertainties caused by the calibration process, since calibration prior to the experiments was not possible and thus the post calibration may not be fully reliable.

\section{HIGH RESOLUTION MEASUREMENTS}

This chapter presents selected examples demonstrating the high spatiotemporal resolution of the diagnostic, as well as the accessible measurement range.

The application of thermal helium beam measurements underlies two major constraints. First, neutral helium is required for line ratio emission spectroscopy. Second, the underlying plasma parameters have to lead to a sufficient excitation of the neutral helium. This limits the application of the diagnostic to a certain parameter range in $n_{\mathrm{e}}$ and $T_{\mathrm{e}}$. This parameter range typically lies in the separatrix and the near SOL region of magnetic fusion devices ${ }^{4}$. In the far SOL, from where helium is injected, the neutral helium density is highest. Here, due to low temperatures, the emission is limited by a too low excitation rate. Further inside, the neutral helium density decreases due to increasing electron temperature and density. These ion- 
ization losses limit the application at higher $n_{\mathrm{e}}$ and $T_{\mathrm{e}}$ values.

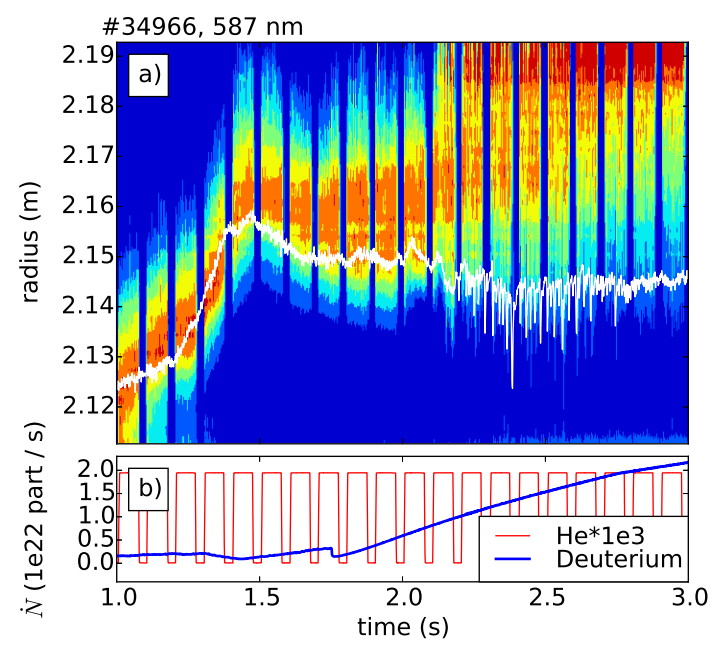

FIG. 12: Contour plot a) showing the $587.6 \mathrm{~nm}$ helium emission along the radius over time during a plasma discharge. The shift of the separatrix position (white

line) and the fueling ramp b) shifts the point of maximal emission along the radius. Subplot b) also shows the sharply chopped helium flux, enabling offset subtraction between the pulses.

This is shown in Figure 12, which shows the total emission of the $587.6 \mathrm{~nm}$ line as a function of time and plasma radius in a contour plot. The radial region with pronounced helium emission has a width of $4-5 \mathrm{~cm}$, following the separatrix position, marked as white line in Figure 12 a). This pronounced response of the signal shows the high radial resolution of the measurement. The fueling ramp shown in b) leads to an increase of the plasma density and thus to a radial outwards shift of the helium emission region.

The transition from the low to the high confinement mode in the presented AUG discharge appears at $2.04 \mathrm{~s}$. In the far SOL during H-mode, the signal is mostly produced by intermittent events created during crashes of edge localized modes (ELMs) or inter-ELM turbulent driven filaments. Both lead to increased radial transport of temperature and density. Whereas these events increase the signal in the far SOL, they lead to a decrease of the signal further inside due to ionization losses further outside.

This can be seen in Figure 13 which also demonstrates the high temporal resolution of the signal. The plot shows four zoom levels of the signal time trace of a channel located at a fixed spatial position at $R=2.14 \mathrm{~m}$. For $t \gtrsim 2.1 \mathrm{~s}$, this location corresponds roughly to the separatrix position. For $t \lesssim 1.7 \mathrm{~s}$, the response of the signal to the aforementioned separatrix shift can be seen.

The overview signal reflects the chopping of the helium beam and fluctuations caused by plasma phenomena. The lower two plots compare the helium emission at the separatrix and the far SOL to the divertor current signal (red), which indicates the occurrence of ELMs. The bottom plot shows an additional radial channel at $R=2.19 \mathrm{~m}$ (purple). As mentioned above, the signal strengths for the two plotted channels behave inversely to each-other. Besides the response to dominant ELMs (which are visible in the divertor current signal), the helium emission is also sensitive to inter-ELM filaments.

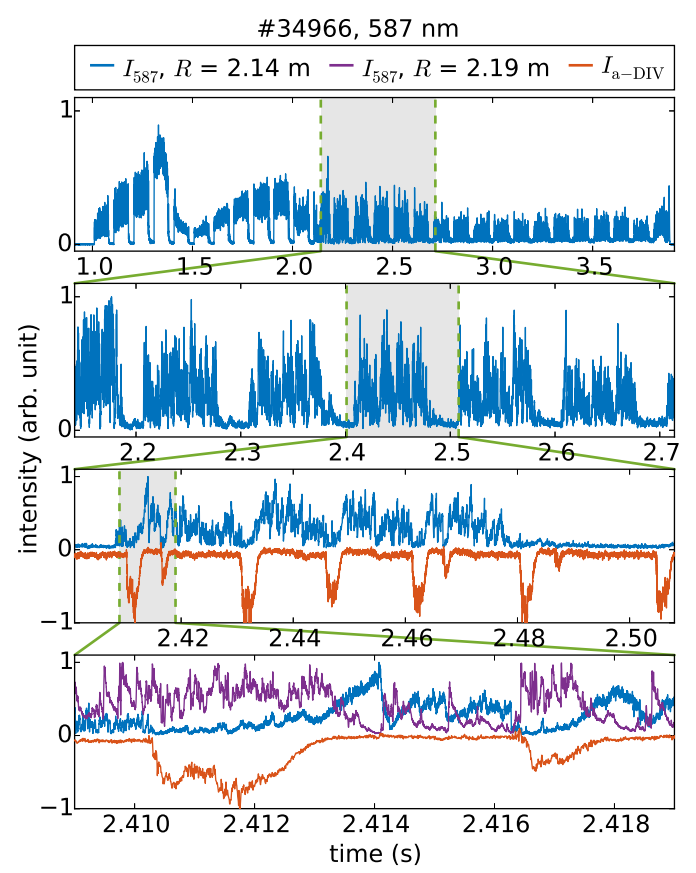

FIG. 13: Time trace of the $587.6 \mathrm{~nm}$ helium line emission during AUG discharge \#34966 with increasing temporal resolution, as measured at $R=2.14 \mathrm{~m}$. The

lower subplots compare the helium emission to the divertor current signal (red). The bottom plot shows an additional radial channel at $R=2.19 \mathrm{~m}$ (purple).

Figure 12 as well as Figure 13 show that the helium injection system ${ }^{5}$ performs well, enabling background subtraction during the beam-off phases. Furthermore, a very good signal-to-noise ratio can be seen. Exemplary plasma profiles for the L-mode phase are shown in Figure 14, with comparison to the data from the Thomson scattering diagnostic. With reservations of possible calibration discrepancies of the helium beam optics, the resulting profiles are in good agreement with the Thomson data. The increase of $T_{\mathrm{e}}$ in the far SOL is a known effect and can be explained by the static collisional radiative model used for the evaluation. This effect can be compensated by a time dependent approach, as described in ${ }^{4,6}$. It has been found that the density profiles agree very well with other diagnostics for a variety of plasma scenarios. The temperature profiles, however, are not always consistent with Thomson scattering measurements and further investigations into their reliability in different scenarios is planned. 

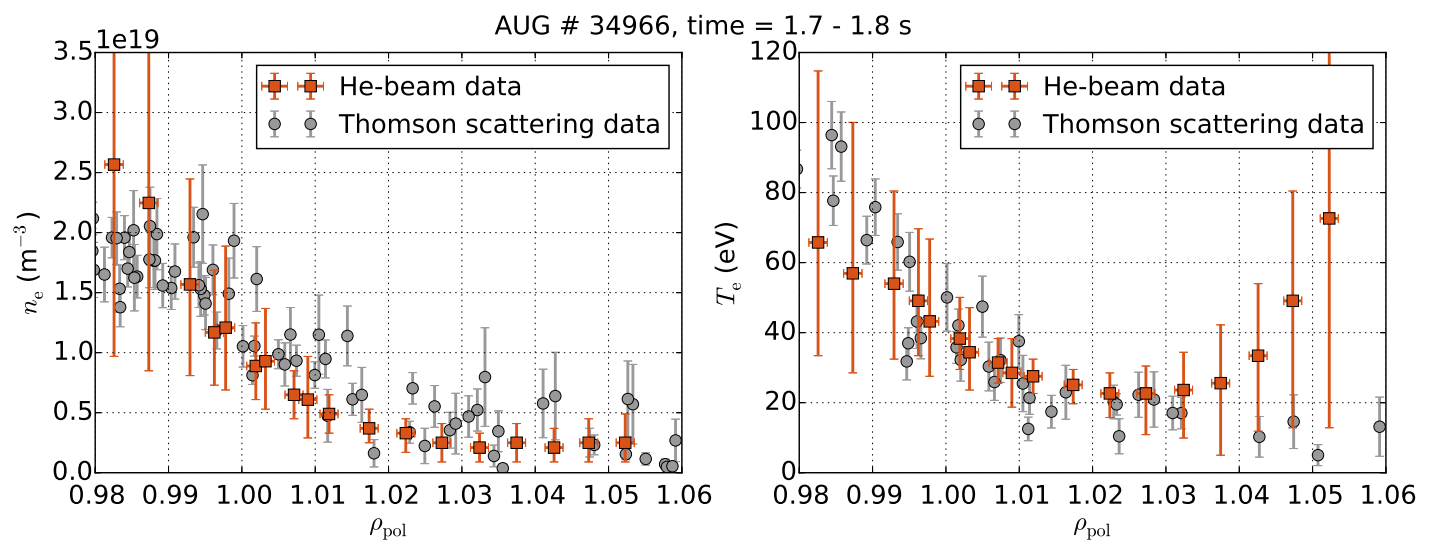

FIG. 14: Radial L-mode profiles of $n_{\mathrm{e}}$ and $T_{\mathrm{e}}$ showing a comparison of the thermal helium beam with the Thomson scattering diagnostics, averaged over $100 \mathrm{~ms}$.

\section{SUMMARY AND CONCLUSION}

A bundle of hardware components is presented which enables spatiotemporal highly resolved line ratio spectroscopy on thermal helium at ASDEX Upgrade. The high spatial resolution of up to $3 \mathrm{~mm}$ is enabled by an optical head containing an innovative clamping mechanism for optical fibers. The signals from 32 fibers can be measured simultaneously with the developed four-color polychromator system, enabling a temporal resolution of $900 \mathrm{kHz}$.

It is demonstrated that the helium beam diagnostic offers fast and spatially highly resolved electron temperature and density measurements that cover the plasma edge and SOL region. While such measurements of the electron density have been available for quite some time, the simultaneous determination of critical transport quantities in $n_{\mathrm{e}}$ and $T_{\mathrm{e}}$ is unique. Single edge localized modes, filaments, and other turbulent structures are resolved, allowing analysis of their influence on $n_{\mathrm{e}}$ and $T_{\mathrm{e}}$.

\section{ACKNOWLEDGMENTS}

This work has been carried out within the framework of the EUROfusion Consortium and has received funding from the Euratom research and training programme 2014-2018 under grant agreement No 633053. The views and opinions expressed herein do not necessarily reflect those of the European Commission.

This work was funded in part by the U.S. Department of Energy under Grant DE-SC00013911 and DESC0014210.

${ }^{1}$ H. Zohm, Nuclear Fusion 55, 104010 (2015).

${ }^{2}$ E. A. Unterberg, O. Schmitz, D. H. Fehling, H. Stoschus, C. C. Klepper, J. M. Muñoz-Burgos, G. Van Wassenhove, and D. L. Hillis, Review of Scientific Instruments 83, 10D722 (2012).

${ }^{3}$ U. Kruezi, H. Stoschus, B. Schweer, G. Sergienko, and U. Samm, Review of Scientific Instruments 83, 065107 (2012).

${ }^{4}$ M. Griener, J. M. M. Burgos, M. Cavedon, G. Birkenmeier, R. Dux, B. Kurzan, O. Schmitz, B. Sieglin, U. Stroth, E. Viezzer, and E. Wolfrum, Plasma Physics and Controlled Fusion 60, 025008 (2018).

${ }^{5}$ M. Griener, O. Schmitz, K. Bald, D. Bösser, M. Cavedon, P. D. Marné, T. Eich, G. Fuchert, A. Herrmann, A. Kappatou, T. Lunt, V. Rohde, B. Schweer, M. Sochor, U. Stroth, A. Terra, and E. Wolfrum, Review of Scientific Instruments 88, 33509 (2017).

${ }^{6}$ J. M. Muñoz Burgos, O. Schmitz, S. D. Loch, and C. P. Ballance, Physics of Plasmas 19, 012501 (2012).

${ }^{7}$ T. Barbui, M. Krychowiak, R. König, O. Schmitz, J. M. M. Burgos, B. Schweer, A. Terra, W.-X. Team, J. Munoz-Burgos, B. Schweer, A. Terra, J. M. Muñoz Burgos, B. Schweer, A. Terra, W.-X. Team, J. M. M. Burgos, B. Schweer, A. Terra, and W.-X. Team, Review of Scientific Instruments 87, 11E554 (2016).

${ }^{8}$ R. J. Colchin, D. L. Hillis, R. Maingi, C. C. Klepper, and N. H. Brooks, Review of Scientific Instruments 74, 2068 (2003).

${ }^{9}$ S. Kajita, N. Ohno, S. Takamura, and T. Nakano, Physics of Plasmas 13, 1 (2006).

${ }^{10}$ D. Dunai, S. Zoletnik, J. Sarközi, and A. R. Field, Review of Scientific Instruments 81 (2010), 10.1063/1.3488458.

${ }^{11}$ M. Agostini, P. Scarin, R. Cavazzana, L. Carraro, L. Grando, C. Taliercio, L. Franchin, and A. Tiso, Review of Scientific Instruments 86, 123513 (2015).

${ }^{12}$ S. J. Zweben, J. L. Terry, D. P. Stotler, and R. J. Maqueda, Review of Scientific Instruments 88, 41101 (2017).

${ }^{13}$ G. Fuchert, G. Birkenmeier, D. Carralero, T. Lunt, P. Manz, H. W. Müller, B. Nold, M. Ramisch, V. Rohde, and U. Stroth, Plasma Physics and Controlled Fusion 56, 125001 (2014).

${ }^{14}$ Asphericon GmbH, Stockholmer Strasse 9, 07747 Jena, Germany.

${ }^{15}$ Art Photonics GmbH, Rudower Chaussee 46, 12489 Berlin, Germany.

${ }^{16}$ Ceram Optec SIA, Skanstes iela 7, 1013 Riga, Latvia.

${ }^{17}$ Opto Engineering Deutschland GmbH, Agnes-Pockels-Bogen 1, 80992 München, Germany.

${ }^{18}$ Laser Components GmbH, Werner-von-Siemens-Straße 15, 82140 Olching, Germany.

${ }^{19}$ Avantes BV, Oude Apeldoornseweg 28, 7333 Apeldoorn, Netherlands.

${ }^{20}$ Hamamatsu Photonics Deutschland GmbH, Arzbergerstr. 10, 82211 Herrsching am Ammersee, Germany.

${ }^{21}$ K. Behler, H. Blank, H. Eixenberger, A. Lohs, K. Lüddecke, R. Merkel, G. Raupp, G. Schramm, W. Treutterer, and M. Zilker, Fusion Engineering and Design 83, 304 (2008).

${ }^{22}$ K. Behler, H. Blank, H. Eixenberger, M. Fitzek, A. Lohs, K. Lüddecke, and R. Merkel, Fusion Engineering and Design 87, 2145 (2012). 